\title{
Perspectives, issues and solutions in RNAi therapy: the expected and the less expected
}

\author{
Achim Aigner*,1 \\ ${ }^{1}$ Rudolf-Boehm-Institute for Pharmacology \& Toxicology, Clinical Pharmacology, University of Leipzig, Faculty of Medicine, Leipzig, \\ Germany \\ *Author for correspondence: Tel.: +49 (0)341 97 24661; Fax: +49 (0)341 97 24669; achim.aigner@medizin.uni-leipzig.de \\ ' In the longer range, siRNA therapeutics may well help revolutionize drug development, \\ especially when looking at otherwise undruggable/incurable pathologies."
}

First draft submitted: 27 August 2019; Accepted for publication: 3 October 2019; Published online: 22 November 2019

Keywords: clinical translation • delivery $\bullet$ nanocarrier $\bullet$ RNAi $\bullet$ siRNA • siRNA target identification

\section{The principle of RNAi \& its advantages for therapeutic application}

20 years after the discovery of RNA interference in the late 1990s [1], it is obvious that RNAi has opened novel avenues toward innovative therapeutic strategies based on nucleic acids. Unlike most other oligonucleotide systems, RNAi relies on a catalytic mechanism and thus requires lower amounts of nucleic acid to be delivered to the cell, and siRNA-based cleavage is more efficient compared with ribozymes. In fact, highly potent siRNAs usually show activity even in picomolar concentrations and the delivery of less than 2000 siRNA molecules per cell was found to be sufficient to exert specific gene knockdown [2]. Also, with early encouraging results on the preclinical in vivo application of RNAi, such as the targeting of Fas to protect mice from fulminant hepatitis [3], it was not surprising that the discovery of the key mechanisms and siRNAs, led to a hype with regard to their translation into the clinics. This is particularly so since in the era of full human genomes and the availability of appropriate in silico siRNA prediction tools, it is relatively easy to find siRNAs against virtually any therapeutic target, despite the still remaining need for extensive experimental validation [4]. This also makes lead optimization a more rapid and straightforward process when compared with other pharmacological compounds. Additionally, siRNAs are relatively easy to synthesize, even on a large scale, which can pose a major issue in the case of biologicals. Based on the post-transcriptional rather than post-translational mechanism, in other words, siRNAs acting on the mRNA rather than on the protein level, it is also possible to target and eventually inhibit otherwise undruggable genes, in other words, those where no protein inhibitors exist or cannot be obtained. From a pharmacological viewpoint, this is particularly noteworthy since only a small fraction of the proteins in the human genome is potentially druggable [5]. This would leave the majority of proteins unavailable for inhibition by classical pharmacological approaches, for example due to the absence of active binding sites on the protein. Also, siRNAs can allow for distinguishing between wild-type genes and their pathological counterparts bearing rather subtle mutations like a single point mutation which could be barely or not at all addressed by an inhibitor on the protein level. Just two examples include the ADO II [6] or KRAS ${ }^{G 12 D}$ genes [7]. Taken together, this allows for the exploration of novel targets through the development of RNAi-based drugs and, provided that key problems are solved, substantial progress in therapy can be anticipated.

Beyond the protein-encoding genome, these knockdown strategies can also work for targeting and inhibiting the noncoding genome (e.g., see $[8,9]$ ). It is now well established that $70-90 \%$ of our genome is transcribed, rather than just the very small fraction of protein-encoding genes, and that non-coding long or short RNAs play major roles in human physiology and pathophysiology [10]. While we are only just beginning to recognize the contributions of long non-coding RNAs, knockdown strategies - and perhaps only those - will allow us to explore therapeutic intervention based on their targeting.

It has been clear for a while that RNAi will definitely be able to lead to novel drugs, albeit its manifestation in clinical reality has started only recently (2018) with the first siRNA drug reaching the market (patisiran). This 
can be considered as a long-awaited milestone in therapeutic siRNA development. Unlike CRISPR-Cas9, RNAi acts on the RNA rather than the DNA level, thus avoiding issues related to genetic safety. On the other hand, it may be put forward that for this reason RNAi suffers from the disadvantage of exerting transient effects. However, knockdown has been shown to last for weeks in nondividing cells, with siRNA 'dilution' through cell division being the most relevant limitation of sustained siRNA efficacy [11]. In contrast, intracellular 'drug instability' does not seem to be a major issue since siRNAs have been found to be remarkably stable in the intracellular environment [12], and the active antisense strand of the siRNA may also be protected by its incorporation into the RNA-induced silencing complex. Intracellular stability is even more enhanced upon introducing chemical modifications to the siRNA oligonucleotide, which have been extensively explored and can be implemented especially on the passenger strand. Over 100 unique chemical RNA modifications allow for fine-tuning of siRNA properties in terms of stability/intracellular half-life and strand bias as well as specificity and efficacy [13]. They have led, for example, to Alnylam's so-called 'enhanced stabilization chemistry' for designing metabolically stable siRNAs or other rather sophisticated patterns of siRNA sequences, containing modified nucleotides at defined positions with various combinations and permutations [14].

Thus, while from a pharmacologist's viewpoint the transient nature of an inhibitor is the normal situation rather than an exception, RNAi may actually last even longer than other inhibitory molecules. In fact, clinical studies on Patisiran administered intravenously only every 3 weeks over 18 months for the treatment of transthyretin (ATTR) amyloidosis, a debilitating and fatal disease caused by TTR amyloid accumulation, demonstrated a sustained reduction of mean serum levels of the target gene, TTR [15]. On the other hand, in rapidly dividing (cancer) cells, the duration of gene knockdown may indeed be governed by cell division, requiring the adaptation of dosing schedules especially for ensuring that the strongly proliferating parts of the tumor tissue receive adequate treatment over time. Finally, in many cases the still transient nature of RNAi can also be seen as an advantage; which drug do we really want to act forever and in which cases is this really a therapeutic requirement?

Despite the many obvious advantages of RNAi, its therapeutic application soon met and had to tackle a rather obvious and long-known problem: delivery.

\section{The still existing issue in RNAi therapy: delivering siRNAs}

In contrast to other nucleic acids, like antisense oligonucleotides, RNA molecules must be considered as even less stable, and beyond their rapid degradation by nucleases leading to a half-life of only a few minutes in blood serum [16], they are negatively charged and still too large $(\sim 13 \mathrm{kD})$ for passing biological barriers and uptake by target cells. Thus, compared with single-stranded antisense oligonucleotides, the delivery of double-stranded siRNAs is even more challenging, particularly in vivo. In fact, 20 years after the discovery of RNAi, the efficient delivery of its effectors, siRNA, still poses the major limitation to their therapeutic use. Viral delivery systems, although highly efficient, are less attractive due to safety and toxicity issues and nonviral systems are preferred.

To some extent this has led to some focusing on local delivery, for example, intravitreal injection [17], or on target organs that are comparatively easy to reach, in particular the liver. The field immensely benefited from previous work on nonviral delivery of oligonucleotides and novel developments in the two decades after the discovery of RNAi. In fact, a wide variety of lipid, polymeric, inorganic and other carriers have been developed for siRNA encapsulation or adsorption and tested in vitro and in vivo, aiming at delivering its payload to the target organ/tissue, facilitated cellular internalization, intracellular release from the endosomal/lysosomal system and siRNA release from its formulation. Since the various systems are extensively reviewed elsewhere (e.g., see [18,19]), they will not be discussed here in greater detail. It should be noted, however, that from the innumerable papers on the various systems, translation into clinical trials has been limited to only a few of those. These include lipid-based nanoparticles (LNPs), especially stable nucleic acid lipid particles as second generation LNPs [20], and siRNA conjugates, namely $\mathrm{N}$-acetylgalactosamine (GalNAc) for high-affinity binding to the asialoglycoprotein receptor, for delivery into hepatocytes [21]. LNPs are not entirely new since various liposome-formulated cytostatic drugs like doxorubine $\left(\right.$ Doxil $\left.^{\circledR}\right)$, daunorubicine $\left(\right.$ DaunoXome ${ }^{\circledR}$ ) or vincristine $\left(\right.$ Marqibo ${ }^{\circledR}$ ) have been approved already (e.g., see [18] for review), and thus can be adapted to delivery challenges in siRNA therapies by LNP design and lipid composition. Issues related to a permanently positive surface charge leading to toxicity and rapid clearance by the reticuloendothelial system have led to second generation systems with $\mathrm{pH}$-dependent ionization. While the first siRNA drug approved relies on a stable nucleic acid lipid particle formulation, GalNAc conjugates [22] are particularly efficient and eventually may perhaps replace many LNP formulations for liver targeting. However, they are limited to hepatocytes, and no siRNA conjugation partners with comparable efficacy in other cell types 
exist. LNPs rely on much defined lipid compositions and the production of LNPs is rather complicated. It can thus be anticipated that other delivery vehicles will be explored even more thoroughly in the near future. These include, but are not limited to polymeric nanoparticles for siRNA complexation, which offer a broad spectrum of chemical modifications for optimizing efficacy and biocompatibility, solid nanoparticles for siRNA transport by adsorption and siRNA conjugation to aptamers, biomolecules or other ligands beyond GalNAc. Combinations between different systems, for example polymeric nanoparticles and liposomes, are feasible as well [23,24]. Of note, biological systems like exosomes or other extracellular vesicles are also explored, for example in metastatic cancer for delivering siRNAs against the G12D mutant form of KRAS [25]. By obtaining exosomes from defined cell types, they may also provide some target cell specificity due to specific binding moieties on their surface.

The concept of targeted delivery, relying on the specific binding of conjugated siRNAs or ligand-decorated nanoparticle systems onto the surface of a target cell, is intriguing also with regard to reaching hard-to-transfect cells, and may in particular provide avenues for targeting defined subsets of immune cells [26]. On the other hand, targeted delivery should not be overestimated since it does not actively guide a given siRNA or nanoparticle to its target tissue, but will only lead to its retention at the preferred site and perhaps aid its cellular internalization. It should also be kept in mind that any delivery system should not be too complicated since sophisticated multicomponent systems will also add regulatory hurdles. Many systems published do not seem to meet this criterion. In conclusion, the development of novel delivery strategies and systems is still a major issue, clinical efforts are definitely relevant beyond local siRNA application or delivery into the liver, and despite the many requirements systems should still be kept as simple as possible.

In this context, it has also become clear that in vitro testing in normal $2 \mathrm{D}$ cell culture is often insufficient for assessing the potential of a given nanocarrier and can thus only be the first step. Even structure-function relationships of nanoparticle modifications may well rely on critical parameters which are poorly or not at all represented in $2 \mathrm{D}$ cell culture, making results incomplete or even misleading. Thus, the development and stringent testing of promising nanosystems heavily relies on powerful 3D in vitro, in vivo and/or ex vivo models, for example, in tumor biology. In this context, it should be also kept in mind that a given nanocarrier with high efficacy, for example, for plasmid DNA may be poor for siRNA delivery, and vice versa. This can be readily explained by the different nature of the nucleic acids, different intracellular target sites and other variables, leading to different nanoparticle requirements. As outlined above, a given nanocarrier needs to meet several important, but sometimes almost mutually exclusive criteria and features: high stability and efficient extracellular protection of the siRNA, but also its efficient intracellular release; high-target cell but low off-target cell uptake, high efficacy but absence of toxicity and other unwanted effects like immunostimulation. With regard to the latter, however, it is interesting to speculate that in the light of recent immunotherapeutic approaches in tumor therapy [27], some nanoparticletriggered immunostimulation may actually be advantageous by supporting immune-mediated antitumor activities. And talking of immune therapies in cancer: it should be kept in mind that nanocarrier-mediated siRNA delivery may not only cover tumor cells since other cell types like macrophages, dendritic cells, $\mathrm{T}$ cells, other immune cells or other stroma cells have emerged as attractive targets in tumor therapy as well [28]. Targeting components of the tumor microenvironment may be particularly attractive but may require other nanoparticle properties.

\section{The perhaps less obvious problem: what to deliver - find the right target}

Given the advantage that siRNAs can be developed essentially against any target of interest, the selection of optimal targets takes a pivotal role in the absence of any restrictions regarding drug availabilities. In tumor biology, these may well include rather novel targets, beyond classical oncogenes, since intervention is not restricted to 'classically' druggable genes or the availability of small molecule inhibitors or inhibitory antibodies. Of note, this may also include the combination of different siRNAs in a single nanoparticle, leading to the parallel knockdown of multiple targets through a single formulation [29]. Comparable with targeted therapies in oncology based on small molecule inhibitors, the preselection of correct patient populations in terms of genetic signatures will critically determine success rates of clinical trials and therapy. In tumor biology, recent studies also indicate that the inhibition or knockdown of a selected target gene may lead to the rapid counter-(up)regulation of other oncogenes [JENKE, BÜCH, AIGNER; UNPUBLISHED DATA]. While this cellular plasticity can lead to an unwanted acquired resistance toward a single inhibition or knockdown, it may also result in a new oncogene dependence. For the therapeutic exploration of this new oncogene addiction as an acquired vulnerability of the tumor, switching to another siRNA may then provide a straightforward approach. In fact, it may be easier to find another siRNA for its use in the same formulation than having to identify and switch to another inhibitor. Hence, it is tempting to speculate that the 
time-dependent adaptation of personalized treatment strategies, for example in cancer, could rely on a nanoparticle formulation containing different siRNAs over time.

Combination strategies can also include combining a siRNA approach with an established treatment modality, for example, based on cytostatic drugs in cancer therapy. As an example, the inhibition of multi-drug resistance by siRNA-mediated knockdown of P-gp/MDR1 while simultaneously delivering a chemotherapeutic in the same (nanoparticle) formulation may be considered as a rather straightforward approach and it has been explored in several preclinical studies [30]. It should be kept in mind, however, that time kinetics may be different, with the onset of RNAi and the concomitant reduction of the target gene on the protein level requiring some time. In these cases, the simultaneous delivery of the cytostatic drug may come too early, since it would have to benefit from an already established target gene knockdown and a concomitant reduction of cellular efflux. Consequently, the development of rather complicated nanoparticle systems for simultaneous dual delivery may not be as productive as expected. On the other hand, a single siRNA-mediated oncogene knockdown may prove insufficient for antitumor therapy and using RNAi for the enhancement of targeted inhibitor efficacy or chemotherapy may thus be more promising and may well lead to the design of novel rational combination therapies.

As described above, noncoding RNAs become available as targets as well. Over the past years, RNA biology has revealed new RNA classes with unexpected functions and mechanisms of action, which profoundly affects physiological and pathophysiological processes and are thus potentially interesting candidates as targets for pharmacological intervention [31]. With ncRNAs being largely inaccessible to 'normal' small molecule inhibitors or other drugs, siRNA approaches may allow to substantially broaden the portfolio of targets for therapeutic intervention. This will require, however, the detailed analysis of the various functions and effects of noncoding genes, and the identification of functionally and therapeutically relevant candidates versus 'innocent bystanders' in a given pathology. Thus, in the future the functional analysis of coding and noncoding genes with regard to their pathological values will become even more important. The availability of RNAi-based knockdown, and even more so of CRISPR-Cas9-based knockout approaches [32], provides powerful screening platforms toward this goal.

\section{The problem to be anticipated: translation from bench to bedside}

The discovery of RNAi soon led to the initiation of first clinical studies, mainly based on the local delivery of siRNAs. For many pharmacological and toxicological reasons (high-siRNA concentration at the site of action, largely absent of unwanted effects on nontarget tissues/organs, less requirements for delivery systems), this approach is preferable, but it is obviously restricted to pathologies amenable to local treatment. Age-related macular degeneration was an early example for clinical siRNA studies. However, most early clinical trials remained unsuccessful due to poor efficacy, the occurrence of nonspecific siRNAs effects, toxicity, stimulation of the innate immune system or other reasons. Systemic delivery is indispensable for the treatment of the majority of diseases and is thus being explored quite extensively as well. Currently, a wide variety of clinical trials based on local delivery or systemic delivery are ongoing (e.g., see [33] for review).

According to PubMed, >100,000 papers on studies involving siRNAs have been published, mainly describing laboratory and preclinical work. Initially starting from very low, the numbers of clinical studies have increased over the last decade. The still very low translation rates indicate that, despite the many advantages outlined above, profound difficulties in siRNA translation remain. These include issues related to the carrier system - efficacy, toxicity, especially hepatotoxicity due to preferential accumulation in the liver, specificity/favorable biodistribution, off-target accumulation, long-term safety, immunogenicity - as well as issues related to siRNA molecule and its biological actions (off-target effects, immunogenicity, long-term safety, off-target accumulation/RNA-induced silencing complex saturation). As outlined above, the identification of optimal target genes may be surprisingly tricky as well, for example in oncology in the light of inter- and intra-tumor heterogeneity and cellular plasticity over treatment time.

\section{Where do we stand now?}

Considering the larger number of ongoing clinical trials, more siRNA drug approvals can be expected within the next years. In the longer range, siRNA therapeutics may well help revolutionize drug development, especially when looking at otherwise undruggable/incurable pathologies. Hopefully, transition times from bench to bedside will become shorter and, after the first successful approvals of siRNA drugs, the barrier for investing in siRNA-based drug will become lower. While it took 20 years since the discovery of RNAi to get the first siRNA drug on the 


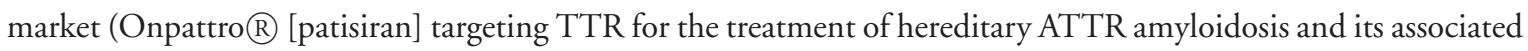
polyneuropathy [34]), more drugs should become available within a shorter time frame.

Upon market introduction, another major issue may be drug costs which are very high. The current US price tag of Onpattro is approximately US $\$ 450,000$ per year, which is explained by high-development costs and comparably small patient numbers ( $\sim 3000$ hATTR patients in the USA). However, it should also be noted that this resembles costs of many other novel therapeutics (small molecule inhibitors, antibodies, in particular immune checkpoint inhibitors) for example in oncology, and costs will become an even bigger issue in the context of cellular therapies (e.g., CAR T-cell therapies). However, at least the manufacturing costs of siRNAs can be expected to be lower as compared with biologicals. Thus, it remains to be seen if siRNA-based therapies will hopefully be able to actually help cap drug expenses.

But independent of the price tag and all other existing issues and limitations, the future looks promising - the first RNAi drug is on the market, with more to come.

\section{Financial \& competing interests disclosure}

The own work of the author and his group on topics covered in this commentary paper was supported by grants from the DFG (Al24/17-1, Al24/21-1), the Wilhelm Sander-Stiftung (2014.099.1; 2015.171.1), the Roland Ernst-Stiftung (06/15) and the Deutsche Krebshilfe (111616). The author has no other relevant affiliations or financial involvement with any organization or entity with a financial interest in or financial conflict with the subject matter or materials discussed in the manuscript apart from those disclosed.

No writing assistance was utilized in the production of this manuscript.

\section{References}

1. Fire A, Xu S, Montgomery MK et al. Potent and specific genetic interference by double-stranded RNA in Caenorhabditis elegans. Nature 391(6669), 806-811 (1998).

2. Wittrup A, Ai A, Liu X et al. Visualizing lipid-formulated siRNA release from endosomes and target gene knockdown. Nat. Biotechnol. 33(8), 870-876 (2015).

3. Song E, Lee SK, Wang J et al. RNA interference targeting Fas protects mice from fulminant hepatitis. Nat. Med. 9(3), 347-351 (2003).

4. Tilesi F, Fradiani P, Socci V et al. Design and validation of siRNAs and shRNAs. Curr. Opin. Mol. Ther. 11(2), 156-164 (2009).

5. Dixon SJ, Stockwell BR. Identifying druggable disease-modifying gene products. Curr. Opin. Chem. Biol. 13(5-6), 549-555 (2009).

6. Maurizi A, Capulli M, Patel R et al. RNA interference therapy for autosomal dominant osteopetrosis type 2. Towards the preclinical development. Bone 110, 343-354 (2018).

7. Zorde Khvalevsky E, Gabai R, Rachmut IH et al. Mutant KRAS is a druggable target for pancreatic cancer. Proc. Natl Acad. Sci. USA 110(51), 20723-20728 (2013).

8. Chen D, Liu L, Wang K et al. The role of MALAT-1 in the invasion and metastasis of gastric cancer. Scand. J. Gastroenterol. 52(6-7), 790-796 (2017).

9. Dong P, Xiong Y, Yue J et al. Long non-coding RNA NEAT1: a novel target for diagnosis and therapy in human tumors. Front. Genet. 9, 471 (2018).

10. Kung JT, Colognori D, Lee JT. Long noncoding RNAs: past, present, and future. Genetics 193(3), 651-669 (2013).

11. Bartlett DW, Davis ME. Insights into the kinetics of siRNA-mediated gene silencing from live-cell and live-animal bioluminescent imaging. Nucleic Acids Res. 34(1), 322-333 (2006).

12. Hoerter JA, Krishnan V, Lionberger TA et al. siRNA-like double-stranded RNAs are specifically protected against degradation in human cell extract. PLoS ONE 6(5), e20352039 (2011).

13. Dar SA, Thakur A, Qureshi A et al. siRNAmod: a database of experimentally validated chemically modified siRNAs. Sci. Rep. 6, 20031 (2016).

14. Foster DJ, Brown CR, Shaikh S et al. Advanced siRNA designs further improve in vivo performance of GalNAc-siRNA conjugates. Mol. Ther. 26(3), 708-717 (2018).

15. Zhang X, Goel V, Attarwala H et al. Patisiran pharmacokinetics, pharmacodynamics, and exposure-response analyses in the Phase 3 APOLLO trial in patients with hereditary transthyretin-mediated (hATTR) amyloidosis. J. Clin. Pharmacol. doi: 10.1002/jcph.1480 (2019) (Epub ahead of print).

16. Layzer JM, McCaffrey AP, Tanner AK et al. In vivo activity of nuclease-resistant siRNAs. RNA 10(5), 766-771 (2004).

17. Rajappa M, Saxena P, Kaur J. Ocular angiogenesis: mechanisms and recent advances in therapy. Adv. Clin. Chem. 50, 103-121 (2010).

18. Wicki A, Witzigmann D, Balasubramanian V et al. Nanomedicine in cancer therapy: challenges, opportunities, and clinical applications. J. Control. Release 200, 138-157 (2015). 
19. Kim B, Park JH, Sailor MJ. Rekindling RNAi therapy: materials design requirements for in vivo siRNA delivery. Adv. Mater. doi: 10.1002/adma.201903637 (2019) (Epub ahead of print).

20. Semple SC, Akinc A, Chen J et al. Rational design of cationic lipids for siRNA delivery. Nat. Biotechnol. 28(2), 172-176 (2010).

21. Rajeev KG, Nair JK, Jayaraman M et al. Hepatocyte-specific delivery of siRNAs conjugated to novel non-nucleosidic trivalent $\mathrm{N}$-acetylgalactosamine elicits robust gene silencing in vivo. Chembiochem 16(6), 903-908 (2015).

22. Springer AD, Dowdy SF. GalNAc-siRNA conjugates: leading the way for delivery of RNAi therapeutics. Nucleic Acid Ther. 28(3), 109-118 (2018).

23. Ewe A, Panchal O, Pinnapireddy SR et al. Liposome-polyethylenimine complexes (DPPC-PEI lipopolyplexes) for therapeutic siRNA delivery in vivo. Nanomedicine 13(1), 209-218 (2016).

24. Schafer J, Hobel S, Bakowsky U et al. Liposome-polyethylenimine complexes for enhanced DNA and siRNA delivery. Biomaterials 31(26), 6892-6900 (2010).

25. Kamerkar S, LeBleu VS, Sugimoto $\mathrm{H}$ et al. Exosomes facilitate therapeutic targeting of oncogenic KRAS in pancreatic cancer. Nature 546(7659), 498-503 (2017).

26. Mei Y, Wang R, Jiang Wet al. Recent progress in nanomaterials for nucleic acid delivery in cancer immunotherapy. Biomater. Sci. 7(7), 2640-2651 (2019).

27. Feng X, Xu W, Li Z et al. Immunomodulatory nanosystems. Adv. Sci. (Weinh.) 6(17), 1900101 (2019).

28. Roma-Rodrigues C, Mendes R, Baptista PV et al. Targeting tumor microenvironment for cancer therapy. Int. J. Mol. Sci. 20(4), 840 (2019).

29. Yu D, Khan OF, Suva ML et al. Multiplexed RNAi therapy against brain tumor-initiating cells via lipopolymeric nanoparticle infusion delays glioblastoma progression. Proc. Natl Acad. Sci. USA 114(30), E6147-E6156 (2017).

30. Tsouris V, Joo MK, Kim SH et al. Nano carriers that enable co-delivery of chemotherapy and RNAi agents for treatment of drug-resistant cancers. Biotechnol. Adv. 32(5), 1037-1050 (2014).

31. Matsui M, Corey DR. Non-coding RNAs as drug targets. Nat. Rev. Drug Discov. 16(3), 167-179 (2017).

32. Wegner M, Diehl V, Bittl V et al. Circular synthesized CRISPR/Cas gRNAs for functional interrogations in the coding and noncoding genome. Elife 8, e42549 (2019).

33. Saw PE, Song EW. siRNA therapeutics: a clinical reality. Sci. China Life Sci. doi: 10.1007/s11427-018-9438-y (2019) (Epub ahead of print).

34. Rizk M, Tuzmen S. Patisiran for the treatment of patients with familial amyloid polyneuropathy. Drugs Today (Barc.) 55(5), 315-327 (2019). 